\title{
Invasive Ductal Breast Carcinoma with Predominant Intraductal Component
}

National Cancer Institute

\section{Source}

National Cancer Institute. Invasive Ductal Breast Carcinoma with Predominant

Intraductal Component. NCl Thesaurus. Code C9132.

A breast carcinoma characterized by the presence of a predominant ductal carcinoma in situ component and a minor component of invasive carcinoma. 